(2) Open Access Full Text Article

REVIEW

\title{
Assessment of adnexal masses using ultrasound: a practical review
}

This article was published in the following Dove Press journal:

International Journal of Women's Health

23 September 2014

Number of times this article has been viewed

\section{Noam Smorgick \\ Ron Maymon}

Department of Obstetrics and Gynecology, Assaf HaRofeh Medical Center, Sackler School of Medicine, Tel Aviv University, Tel Aviv, Israel
Correspondence: Noam Smorgick Department of Obstetrics and Gynecology, Assaf HaRofeh Medical Center, Tzrifin 70300, Israel

Tel +97289779000

Fax +972 89778026

Emailnoam_yossi@yahoo.com
Abstract: Pelvic ultrasound is commonly used as part of the routine gynecologic exams, resulting in diagnosis of adnexal masses, the majority of which are functional or benign. However, due to the possible complications involving benign adnexal cysts (ie, adnexal torsion, pelvic pain) and the utmost importance of early diagnosis and treatment of ovarian cancer, the correct ultrasound diagnosis of adnexal masses is essential in clinical practice. This review will describe the typical ultrasound appearance of the common physiologic, benign, and malignant adnexal masses with the aim of aiding the clinician to reach the correct diagnosis.

Keywords: adnexal cyst, Doppler, ovarian cancer

\section{Introduction}

Pelvic ultrasonography to visualize the adnexa and the uterus is commonly performed in symptomatic and asymptomatic women of reproductive and menopausal age. Although pelvic ultrasound is highly sensitive in detecting adnexal masses, its specificity in detecting malignancy is lower. In addition, the differentiation between functional ovarian masses that will resolve over time and nonfunctional masses has tremendous implications for patients' counseling and management. Other types of adnexal cysts (such as endometrioma, mature cystic teratoma, and paraovarian cysts) are also important to diagnose correctly since they may affect patients' fertility, may be associated with significant pelvic disease, or put the patient at risk for ovarian torsion. ${ }^{1}$ Thus, the correct use of pelvic ultrasonography has become an integral part of the gynecologic evaluation and exam. The current review will summarize the main ultrasound findings for the most common adnexal masses, with an emphasis on the practical diagnosis of different cyst types.

\section{Clinical assessment}

Although not directly within the scope of this review, the clinical assessment of the patients undergoing evaluation for adnexal masses is of the utmost importance in guiding management: conservative follow-up with timed repeat scans versus surgical intervention. The first clinical parameter to be considered is the patients' age: while adnexal cysts are the most common in reproductive-age women, the likelihood of malignancy in this age group is low, and a large proportion of cysts are of functional origin, tending to resolve over time. On the other hand, in postmenopausal women, the risk of malignancy and therefore clinical suspicion for malignancy are higher. Other factors to consider when evaluating patients with adnexal masses are: symptoms of 
pelvic pain (which may point to adnexal torsion but also to endometriosis, pelvic inflammatory disease, or an acutely hemorrhagic corpus luteum cyst); abdominal distention accompanied by gastrointestinal complaints and weight loss (which may arise from an advanced ovarian malignancy); and use of hormonal contraception (which may affect the likelihood of functional ovarian cysts). In addition, personal or family history of breast and/or ovarian cancer as well as known carrier state for the BRCA 1 or 2 genes will likely direct clinical management towards a less conservative approach.

\section{Simple cyst}

Simple cysts are readily identified on grayscale ultrasound by their unilocular appearance and lack of cyst wall papillae (Figure 1). Further investigation should be undertaken of the cyst's diameters, since small simple cysts, usually less than 2.5-3 cm, are of little clinical importance in reproductiveage women. ${ }^{1}$ Simple cysts are very common and comprise a wide range of pathologies, from the self-limited follicular cysts which will resolve spontaneously upon follow-up of several months, to benign persistent cysts of epithelial origin (most commonly serous cystadenoma), to the very rare case of malignancy. Cases of malignancy in simple cysts are rare in all age groups; however, in those cases where malignancy was diagnosed in a seemingly simple cyst, demographic and clinical risk factors for ovarian cancer were present, such as postmenopausal status and a personal history of breast cancer or ovarian cancer. ${ }^{2}$ Moreover, cases of malignancy in a simple cyst usually involve large cysts $(>7.5 \mathrm{~cm}$ in diameter $){ }^{3}$

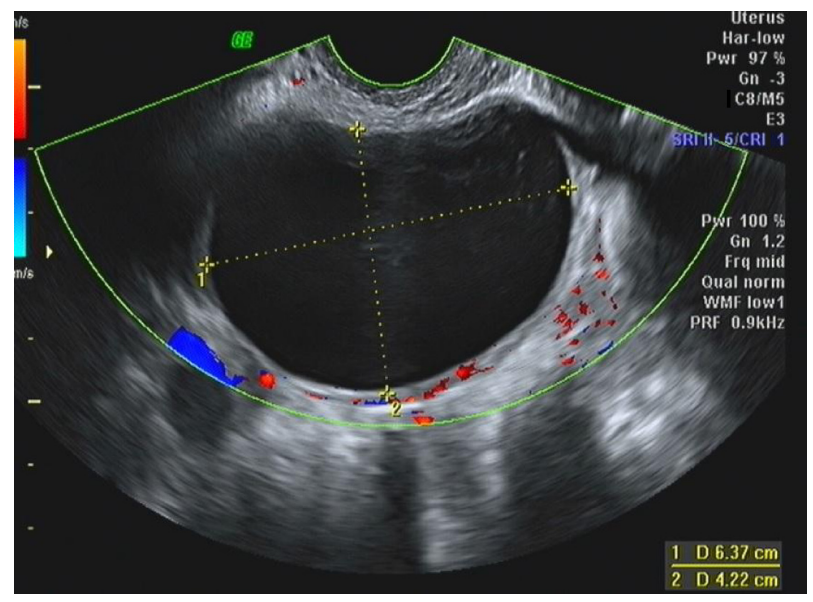

Figure I Transvaginal ultrasound in a 25-year-old woman.

Notes: This simple cyst measuring $64 \mathrm{~mm}$ by $42 \mathrm{~mm}$ was seen on transvaginal ultrasound in a 25 -year-old woman complaining of lower abdominal pain. She was followed for several months with cyst persistence and therefore underwent laparoscopic cystectomy. On laparoscopy, a smooth walled cyst containing clear citrine fluid was seen. Pathology revealed a benign cystadenoma.
In many of those cases where malignancy was diagnosed in a simple cyst, the macroscopic examination of the cyst did reveal nodules in the cyst wall, prompting the suggestion that these cysts were not truly "simple" and that a more detailed preoperative ultrasound examination may have revealed those complex features. ${ }^{2}$

\section{Hemorrhagic cyst}

The most common type of hemorrhagic cyst occurs from bleeding within a corpus luteum cyst. In reproductive-age women, the corpus luteum cysts are functional cysts that resolve upon conservative follow-up, and are diagnosed in symptomatic women with acute pelvic pain or in asymptomatic women. The grayscale ultrasound appearance of an acute hemorrhagic corpus luteum cyst is of cyst with echogenic content which may appear homogeneous or heterogeneous. In case of ruptured hemorrhagic corpus luteum cyst, free pelvic fluid may be observed. Subsequently, when the clot within the cyst is retracting, the cyst appears as a hypoechogenic cyst with an echogenic structure inside it representing the blood clot (Figure 2). This echogenic structure typically moves with transducer ballottement. Finally, a resolving corpus luteum cyst (a process involving hemolysis of the clot and formation of fibrin strands) appears as an avascular cyst containing irregular fine lines resembling a "cobweb", "reticular pattern", or "lace-like pattern" (Figure 2). ${ }^{4}$ This reticular pattern may be confused with septations, thus raising suspicion for malignancy. However, the reticular pattern seen in a resolving hemorrhagic corpus luteum cyst is different from the septations of a suspicious cyst in several crucial points: the former contain thin lines which do not extend

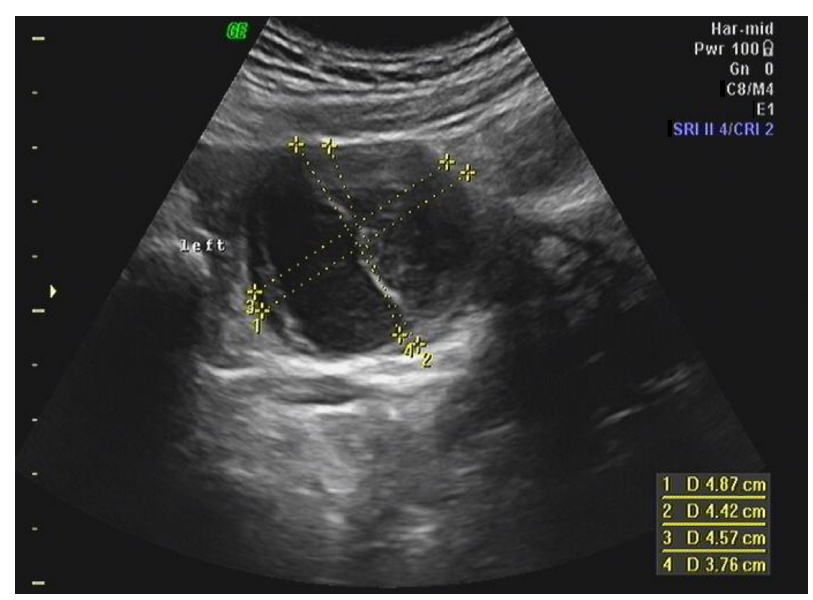

Figure 2 Transabdominal ultrasound in a 16-year-old adolescent.

Notes: A hemorrhagic cyst consistent with a corpus luteum cyst diagnosed on transabdominal ultrasound in a 16-year-old adolescent who presented with acute abdominal pain. A hypoechogenic cyst with an echogenic structure representing the blood clot is observed. In addition, the delicate "cobweb" is seen. 
across the entire cyst diameter, whereas the latter contains thicker lines which extends to the opposite cyst wall. All in all, the classic ultrasound signs detailed above enable an accurate diagnosis of the hemorrhagic corpus luteum cyst in most cases. ${ }^{5}$ Since corpus luteum cysts occur only in reproductive-age women, the appearance of a hemorrhagic cyst in a menopausal woman cannot be due to a functional cyst and often prompts surgical investigation.

\section{Benign cystic teratoma (dermoid cyst)}

Benign cystic teratoma, also called dermoid cysts, are the most common type of germ cell tumors, most often diagnosed in adolescents and reproductive-age women. Because these cysts contain sebaceous material and sometimes hair, their appearance on grayscale ultrasound is of a hyperechoic mass producing an acoustic shadow, ie, gradual attenuation of the sound and obscuring of the structures beyond the cyst (Figure 3). Occasionally, these cysts contain mostly sebaceous fluid, seen on ultrasound as a hypoechoic cyst with echogenic wall components which represent a mixture of hair and more solid sebaceous material (Figure 4). In addition, in those cases where the hair component of the cyst disperses into the cystic fluid, the ultrasound picture is of fine hyperechoic lines called "dermoid mesh". ${ }^{6}$ When the cyst contains bone or teeth, these may also appear as a solid hyperechoic part of the cyst. Despite the diverse appearance of dermoid cysts on ultrasound, their diagnosis is often straightforward, reaching a sensitivity of $99 \% .^{7}$ Nevertheless, dermoid cysts are sometimes difficult to differentiate on ultrasound from hemorrhagic cysts and endometriomas. In those cases,

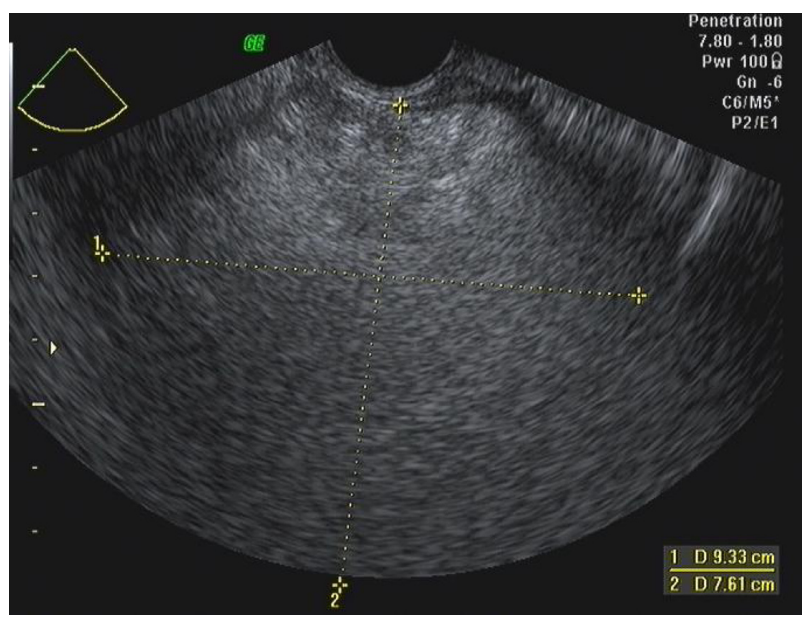

Figure 3 Transvaginal ultrasound in a 70-year-old woman.

Notes: A $90 \mathrm{~mm}$ dermoid cyst diagnosed on routine transvaginal ultrasound in a 70-year-old woman. The cyst contains mostly echoic material and produces a noticeable acoustic shadow with attenuation of the sound beyond the cyst.

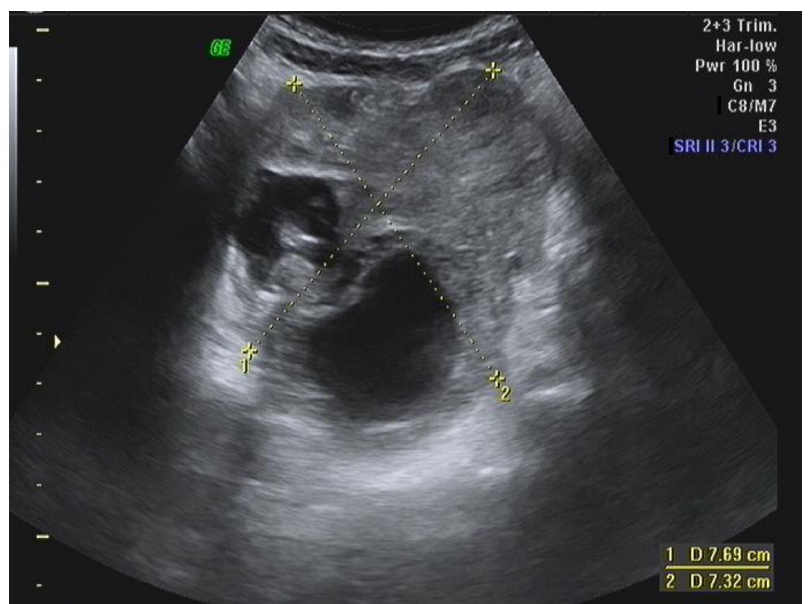

Figure 4 Transabdominal scan in a 9-year-old girl.

Notes: An enlarged ovary was seen on transabdominal scan in a 9-year-old girl who presented with abdominal pain. The ovary contained two cystic areas, one with an echoic structure. In addition, the stroma of the ovary appears edematous and the normal follicular structure is lost. On laparoscopy, torsion of the ovary involving a large dermoid cyst was diagnosed.

computed tomography or magnetic resonance imaging may help reach an accurate diagnosis. ${ }^{8}$

Benign cystic teratoma may harbor malignancy in rare cases (estimated to occur in $0.17 \%$ to $0.3 \%$ of cases). These malignancies are almost always diagnosed on pathology and as yet there are no known preoperative ultrasound grayscale or Doppler flow features that may confidently suggest this diagnosis. However, from a clinical standpoint, suspicion of malignancy in this setting should arise when a benign cystic teratoma is visualized in peri- or postmenopausal patients, and when the diameter of the cyst is large $(>10 \mathrm{~cm}){ }^{9}$

\section{Hydrosalpinx}

Hydrosalpinx represents fluid trapped in a distended fallopian tube with distal occlusion, and occurs in the setting of previous pelvic inflammatory disease. The appearance on grayscale ultrasound is of a tubular and elongated cystic mass with incomplete septations or indentations along its walls ("waist sign" or "cogwheel"). ${ }^{8}$ In order to fully appreciate the tubular structure of the cyst, the ultrasound probe may be turned by a $90^{\circ}$ angle, and a seemingly simple cyst will appear to be tubular (Figure 5). In the chronic stage, small mural nodules may be noted, resembling "beads on string". Those typical pattern are highly suspicious for the diagnosis of hydrosalpinx..$^{10,11}$

\section{Paratubal cyst}

Paratubal cysts, also called paraovarian cysts, typically appear on grayscale ultrasound as unilocular, thin-walled cysts with smooth margins and anechoic contents. In order 


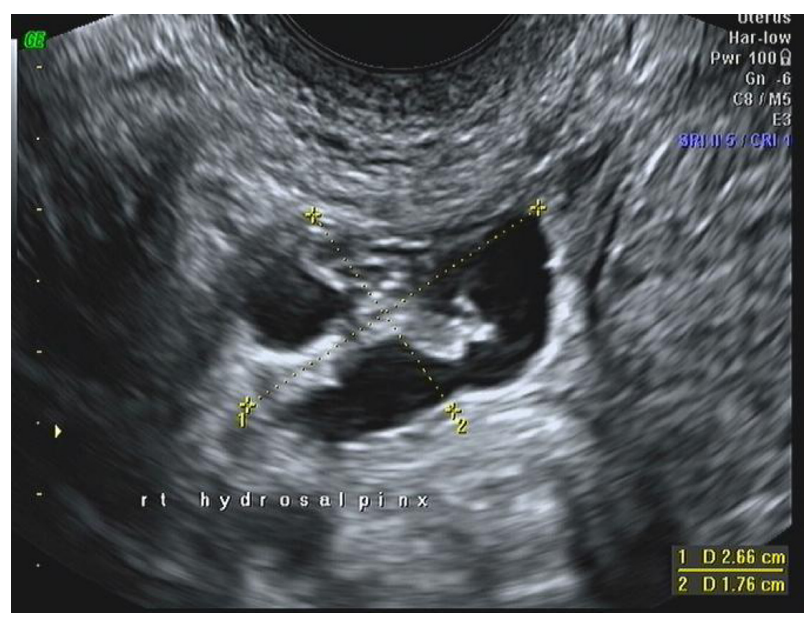

Figure 5 Transvaginal ultrasound in a 28-year-old nulligravida.

Notes: A tubular hypoechoic mass with indentations along it walls consistent with a hydrosalpinx was seen on transvaginal ultrasound in a 28-year-old nulligravida with known tubal occlusion on hysterosalpingogram. Laparoscopy confirmed these findings along with severe pelvic adhesions.

to differentiate these cysts from ovarian simple cysts it is necessary to visualize the ipsilateral ovary separately from the cyst. ${ }^{12}$ Often, these cysts grow to a large size before their diagnosis, and their side localization (ie, right or left) may be difficult. Very rarely, a borderline or overt malignancy may be found in a paratubal cyst, usually in the older reproductive age or perimenopausal age groups. ${ }^{13}$ Suspicious ultrasound findings in cases of paratubal cyst malignancies include papillary projections growing from the cyst wall. ${ }^{14}$ Nevertheless, papillary wall projections may also be seen in cases of benign paratubal neoplasms. Benign paratubal cysts are one of the most common adnexal cysts in adolescents, where they can present with acute pelvic pain due to adnexal torsion. ${ }^{15}$

\section{Endometrioma}

Those "chocolate fluid" filled cysts represent the involvement of the ovaries in the process of endometriosis. Endometriomas have a typical appearance on grayscale ultrasound, as unior multiloculated cysts containing diffuse low-level homogenous echoes, also known as "ground glass" appearance (Figure 6). ${ }^{16}$ However, this typical appearance is present in about $85 \%-90 \%$ of surgically confirmed cases, while, in the remaining, a nontypical appearance is present with cyst wall projections (thought to represent blood clots), heterogeneous appearance of the internal echoes, or even a solid appearance (possibly in chronic ovarian endometriomas). ${ }^{8}$ Thus, a differential diagnosis may exist with hemorrhagic cysts, mucinous cystadenoma, or even malignancy. Use of Doppler flow does not increase the diagnostic accuracy of grayscale ultrasound for the diagnosis endometrioma, since resistance

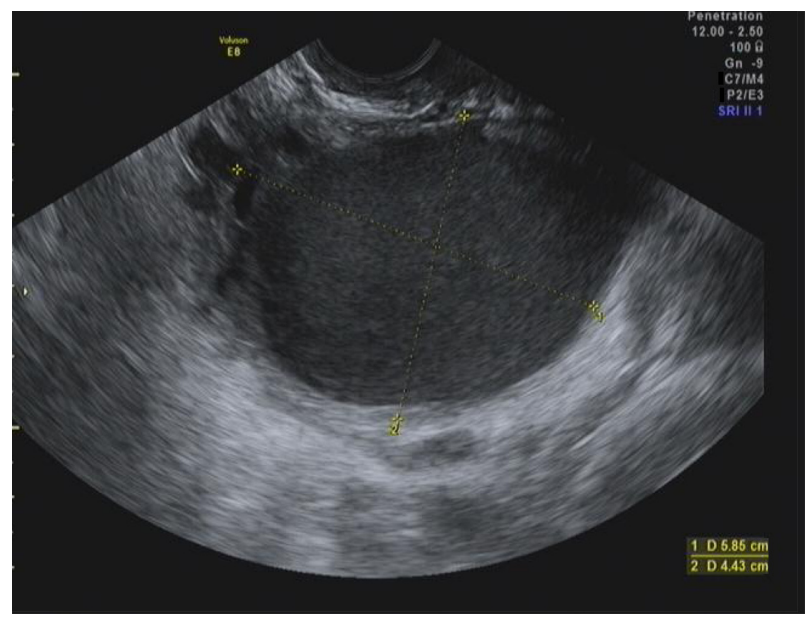

Figure 6 Ovarian cyst observed on transvaginal ultrasound in a 25-year-old woman. Notes: A $58 \times 44 \mathrm{~mm}$ ovarian cyst was observed on transvaginal ultrasound in a 25 -year-old woman who presented with pelvic pain. The "ground glass" typical appearance of endometrioma is noticed. Laparoscopy confirmed the diagnosis.

indices are in the normal range, and color Doppler reveals flow only in the cyst's wall. ${ }^{17}$

\section{Tubo-ovarian abscess (TOA)}

TOAs result from a severe pelvic inflammatory disease and represent a breakdown of the adnexal structures (ie, ovary and fallopian tube) by the infection and inflammation process. The ultrasound appearance of TOAs is variable and depends on the duration of the infection. Over time, as the abscess "matures", part of its content may appear cystic. The recognition of cystic areas in TOAs is important from a clinical standpoint, since those cases may be amenable to percutaneous drainage. Otherwise, the TOA appears as a complex cyst with thick walls and seemingly solid areas. ${ }^{18}$ At times, an adjacent pyosalpinx may be observed. The clinical presentation is the key to the correct diagnosis of TOA.

\section{Peritoneal inclusion cysts}

Inclusion cysts, also called pseudocysts, commonly occur in the setting of previous pelvic surgeries, previous pelvic inflammatory disease, or advanced stage endometriosis. The pseudocysts represent fluid trapped between peritoneal adhesions, and therefore have no actual cyst wall. Thus, the shape of the pseudocyst appears irregular as it is defined by the surrounding structures and adhesions. ${ }^{19}$ Often, the ovary is visualized separately from the cyst but in close proximity to it. It is clinically important to suspect the presence of pseudocysts in the appropriate clinical setting since further surgical intervention is unnecessary and may involve injury to nearby pelvic structures due to pelvic adhesive disease. 


\section{Adnexal torsion}

Adnexal torsion occurs mostly in premenarchal and reproductive-age women, and may involve an adnexal cyst (either ovarian or paratubal) or an otherwise normal adnexa (also called "torsion of normal adnexa"). In the clinical setting of acute pelvic pain often accompanied by nausea and vomiting, and tenderness on abdominal and adnexal palpation, the ultrasound characteristics of adnexal torsion are helpful in reaching the presumed diagnosis of torsion. Those characteristics include either an enlarged ovary with peripheral follicles (thought to represent the stromal edema) or an enlarged ovary with a seemingly solid appearance (Figures 4 and 7). The latter picture is more typical of a longer ischemic process. ${ }^{20}$ Often, free pelvic fluid is noted near the adnexa. When an adnexal cyst is the cause of torsion, it is easily visualized as well and its nature may be determined (ie, dermoid cyst, paratubal cyst, or a hemorrhagic cyst). Use of Doppler flow may be misleading in the diagnosis of the torsion due to high false-negative rate - a torsed adnexa may still be seen as having normal Doppler flows due to the ovary's double blood supply (ie, from the ovarian vessels and the utero-ovarian vessels).

\section{Identification of malignant masses and risk stratification}

Although cysts containing malignant neoplasms of epithelial origin are rare, their timely diagnosis is of the utmost importance since early diagnosis and treatment of ovarian cancer is the most important factor in determining survival. Ultrasound features suggestive of epithelial malignancy include thick septations ( $>2-3 \mathrm{~mm}$ in width), solid components, and cyst wall

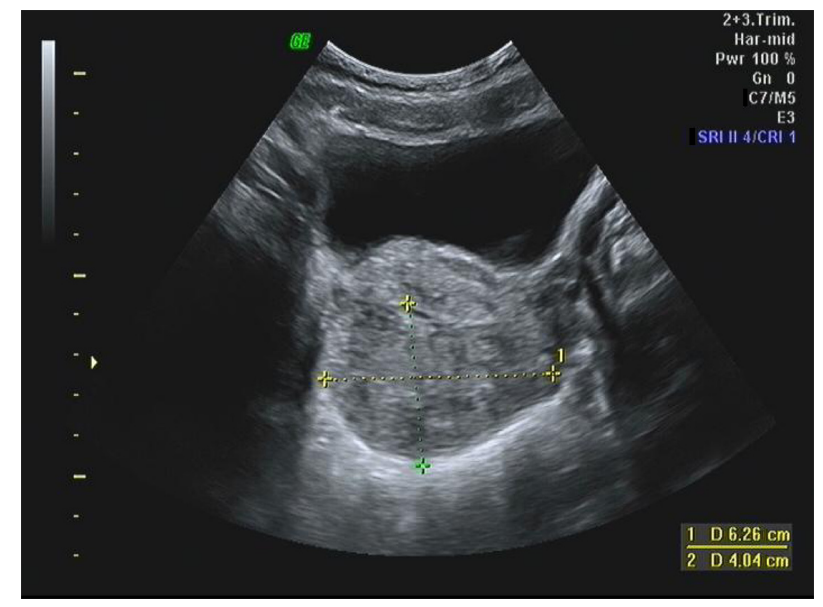

Figure 7 Transabdominal ultrasound scan in an 8-year-old girl with abdominal pain. Notes: An enlarged ovary with loss of follicular structure was seen in an 8-year-old girl who presented with pelvic pain and vomiting. Laparoscopy confirmed torsion of the adnexa. thickening (Figures 8 and 9). The solid areas (or hyperechoic areas) may vary in size, from small nodules or papillations to larger areas. The diameter of the mass appears to be less predictive of malignancy than the features described above. Moreover, malignancies have been described even in relatively small cysts of 3-4 cm in diameter. ${ }^{21}$

The addition of Doppler flow measurements to the grayscale parameters described above may provide additional information in suspicious cases, and has been thought to increase the sensitivity, specificity, and positive predictive value of ultrasound in diagnosing ovarian malignancy. This modality is used to detect abnormal blood vessels which arise from the neovascularization process induced by the malignant lesion. These blood vessels are characterized by abnormal blood flow patterns, typically low resistance to flow, which translates to abnormal pulsed Doppler parameters. However, despite initial interest in this feature, studies have failed to show a significant improvement in detection of malignancy over traditional morphological assessment. The best approach to the correct diagnosis of malignancy now appears to be a combined assessment of gray scale morphologic features and color Doppler imaging. For example, color Doppler may reveal flow within solid areas of the mass, raising suspicion for malignancy. Nevertheless, there is probably a significant overlap between benign and malignant masses in terms of their Doppler flow features. ${ }^{22}$

Three-dimensional ultrasound and three-dimensional power Doppler ${ }^{23,24}$ are relatively new technologies used to assess adnexal masses. Three-dimensional ultrasound visualizes the adnexa in three planes (coronal, sagittal, and frontal) and allows for reconstruction and further analysis of the

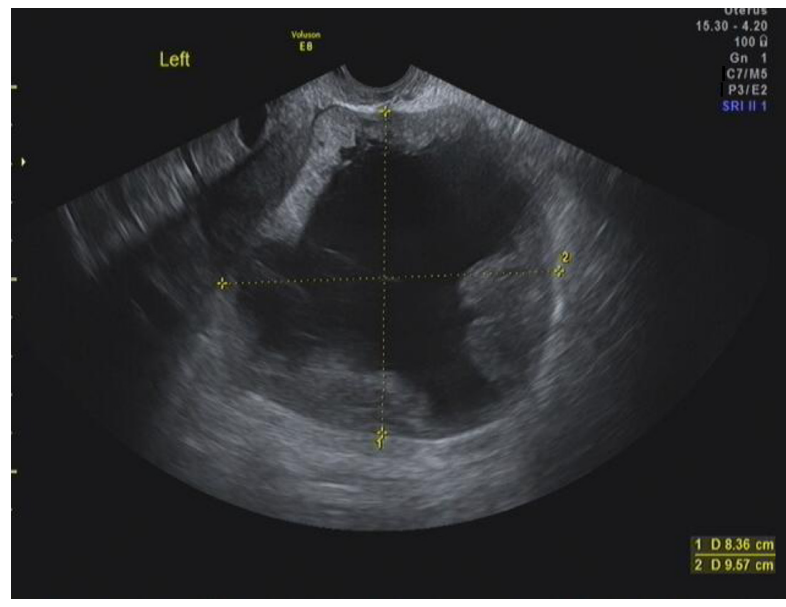

Figure 8 Transvaginal ultrasound in a 64-year-old woman with pelvic mass. Notes: A pelvic mass measuring $83 \times 95 \mathrm{~mm}$ and containing septations and papillations was seen in a 64-year-old woman. Surgery revealed an adenocarcinoma of the ovary. 


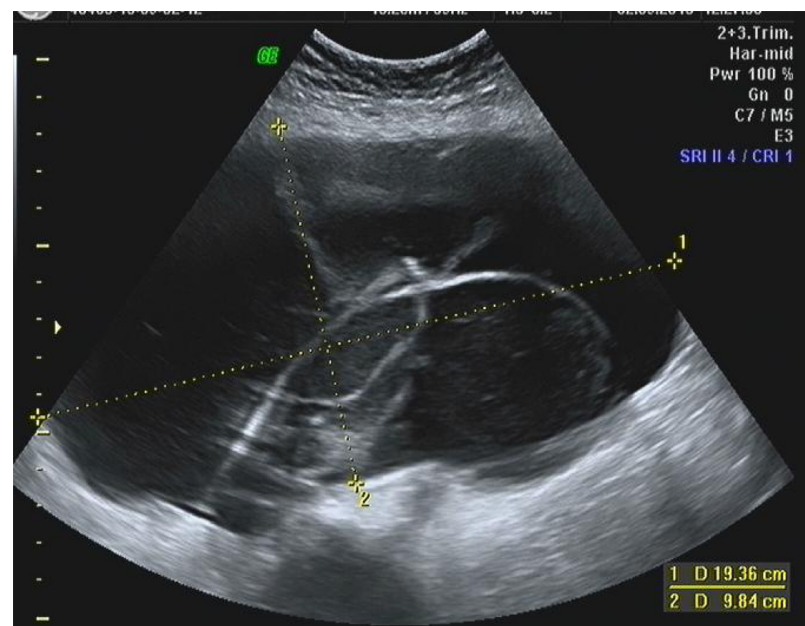

Figure 9 Transabdominal ultrasound scan in a $4 \mathrm{I}$-year-old woman.

Notes: This large cyst in a $4 \mathrm{l}$-year-old woman contains thin septations without increased Doppler blood flow. Surgery revealed a benign mucinous cystadenoma.

volumes acquired and stored, while three-dimensional power Doppler allows for assessment of the vascularity of the mass in all three planes. Findings on three-dimensional ultrasound and power Doppler which have been associated with malignancy include vascular flow in the center of the mass ("central flow"), blood flow within septations and excrescences, and a complex appearance of the vascular architecture. Although current studies have not shown a definite advantage of the three-dimensional power Doppler over two-dimensional power Doppler in accurately diagnosing ovarian malignancy, future studies my help define the role of these technologies in the workup of adnexal masses.

With the aim of increasing the accuracy of ultrasound in the detection of ovarian malignancy, several risk-stratification models have been suggested. ${ }^{25}$ These models ascribe different scores to suspicious ultrasound features and clinical factors (such as age, menopausal status, and CA-125 level). The combination of individual scores provides a final score which should direct the clinician towards conservative follow-up versus surgical intervention. However, when the diagnostic performance of risk-stratification models was compared to "pattern recognition" (ie, subjective evaluation of grayscale and Doppler flow features), the latter actually performed better, yielding a sensitivity of around $85 \%$ and specificity of around $90 \%{ }^{26}$ Thus, the sonographer's experience combined with the appropriate clinical investigation appears to provide the best management to patients with suspicious adnexal masses.

Magnetic resonance imaging (MRI) may be used as an adjunct imaging modality when the initial ultrasound characterization of an adnexal mass as benign or malignant

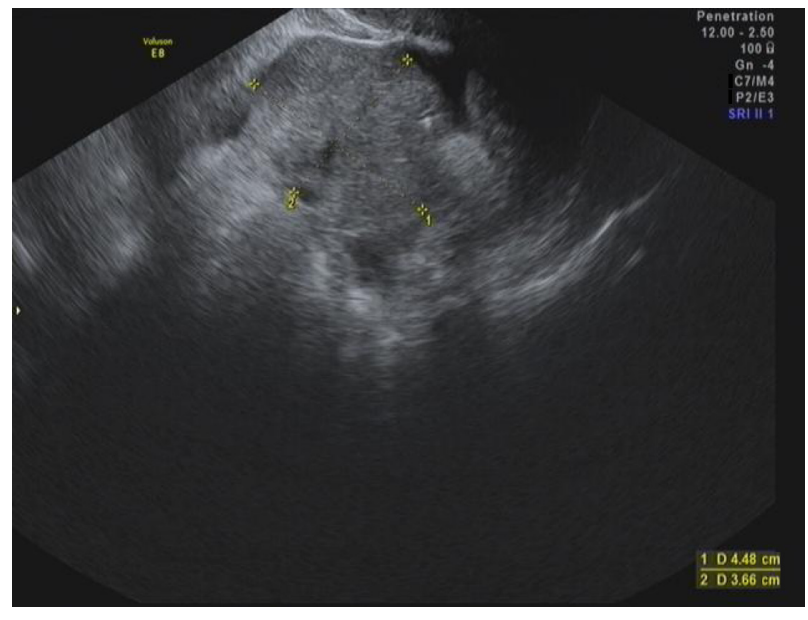

Figure 10 Transvaginal ultrasound scan of a 59-year-old woman.

Notes: The ovary of a 59-year-old woman contains a large solid mass. Surgery revealed this mass to be a benign fibrothecoma.

is inconclusive. A recent meta-analysis found that the sensitivity and specificity of MRI for correct detection of malignancy may reach $92 \%$ and $88 \%$, respectively. ${ }^{27}$ However, the cost of MRI studies and their (sometimes) limited availability should be taken into account as well when planning the patient's workup. Furthermore, in most clinical scenarios, an ultrasound exam performed by an experienced sonographer may provide sufficient information upon which to counsel patients whether or not surgical investigation of the adnexal mass is necessary. Thus, in clinical practice, MRI may provide further reassurance regarding the benign nature of an adnexal mass, based on its reliable diagnosis of benign adnexal masses.

Additional histologic types of ovarian neoplasms include the sex cord stromal tumors (ie, granulosa cell tumors, Sertoli-Leydig cell tumors, and fibrothecoma). These tumors may produce hormones (estrogens or androgens, depending on the histology), so that the clinical presentations may vary from vaginal bleeding to systemic signs of virilization. The fibrothecoma tumors appear as solid masses on ultrasound, often confused with a pedunculated subserous fibroid (Figure 10).

\section{Conclusion}

Use of grayscale ultrasound combined with Doppler measurements when necessary allows the experienced sonographer to reliably diagnose functional, benign, and malignant adnexal masses. ${ }^{28}$ The information obtained from the pelvic ultrasound, combined with patient's history and gynecologic exam, will guide recommendations from treatment, primarily the decision for conservative follow-up versus surgery. 


\section{Disclosure}

The authors report no conflicts of interest in this work.

\section{References}

1. Patel MD. Pitfalls in the sonographic evaluation of adnexal masses. Ultrasound Q. 2012;28:29-40.

2. Valentin L, Ameye L, Franchi D, et al. Risk of malignancy in unilocular cysts: a study of 1148 adnexal masses classified as unilocular cysts at transvaginal ultrasound and review of the literature. Ultrasound Obstet Gynecol. 2013;41:80-89.

3. Ekerhovd E, Wienerroith H, Staudach A, Granberg S. Preoperative assessment of unilocular adnexal cysts by transvaginal ultrasonography: a comparison between ultrasonographic morphologic imaging and histopathologic diagnosis. Am J Obstet Gynecol. 2001;184:48-54.

4. Jain KA. Sonographic spectrum of hemorrhagic ovarian cysts. J Ultrasound Med. 2002;21:879-886.

5. Patel MD, Feldstein VA, Filly RA. The likelihood ratio of sonographic findings for the diagnosis of hemorrhagic ovarian cysts. J Ultrasound Med. 2005;24:607-614.

6. Outwater EK, Siegelman ES, Hunt JL. Ovarian teratomas: tumor types and imaging characteristics. Radiographics. 2001;21:475-490.

7. Mais V, Guerriero S, Ajossa S, Angiolucci M, Paoletti AM, Melis GB. Transvaginal ultrasonography in the diagnosis of cystic teratoma. Obstet Gynecol. 1995;85:48-52.

8. Brown DL. A practical approach to the ultrasound characterization of adnexal masses. Ultrasound Q. 2007;23:87-105.

9. Park JY, Kim DY, Kim JH, Kim YM, Kim YT, Nam JH. Malignant transformation of mature cystic teratoma of the ovary: experience at a single institution. Eur J Obstet Gynecol Reprod Biol. 2008;141: 173-178.

10. Patel MD, Acord DL, Young SW. Likelihood ratio of sonographic findings in discriminating hydrosalpinx from other adnexal masses. AJR Am J Roentgenol. 2006;186:1033-1038.

11. Guerriero S, Ajossa S, Lai MP, et al. Transvaginal ultrasonography associated with colour Doppler energy in the diagnosis of hydrosalpinx. Hum Reprod. 2000;15:1568-1572.

12. Savelli L, Ghi T, De Iaco P, Ceccaroni M, Venturoli S, Cacciatore B. Paraovarian/paratubal cysts: comparison of transvaginal sonographic and pathological findings to establish diagnostic criteria. Ultrasound Obstet Gynecol. 2006;28:330-334.

13. Vaysse C, Capdet J, Mery E, Querleu D. Paratubal endometrioid cystadenocarcinoma: case report and review. Eur J Gynaecol Oncol. 2009;30:443-445.

14. Smorgick N, Herman A, Schneider D, Halperin R, Pansky M. Paraovarian cysts of neoplastic origin are underreported. JSLS. 2009; 13: 22-26.
15. Thakore SS, Chun MJ, Fitzpatrick K. Recurrent ovarian torsion due to paratubal cysts in an adolescent female. J Pediatr Adolesc Gynecol. 2012;25:e85-e87.

16. Asch E, Levine D. Variations in appearance of endometriomas. J Ultrasound Med. 2007;26:993-1002.

17. Alcázar JL, Laparte C, Jurado M, López-García G. The role of transvaginal ultrasonography combined with color velocity imaging and pulsed Doppler in the diagnosis of endometrioma. Fertil Steril. 1997;67:487-491.

18. Varras M, Polyzos D, Perouli E, Noti P, Pantazis I, Akrivis Ch. Tubo-ovarian abscesses: spectrum of sonographic findings with surgical and pathological correlations. Clin Exp Obstet Gynecol. 2003;30: $117-121$.

19. Guerriero S, Ajossa S, Mais V, Angiolucci M, Paoletti AM, Melis GB. Role of transvaginal sonography in the diagnosis of peritoneal inclusion cysts. J Ultrasound Med. 2004;23:1193-1200.

20. Smorgick N, Maymon R, Mendelovic S, Herman A, Pansky M. Torsion of normal adnexa in postmenarcheal women: can ultrasound indicate an ischemic process? Ultrasound Obstet Gynecol. 2008;31: 338-341.

21. van Nagell J, DePriest P, Reedy M, et al. The efficacy of transvaginal sonographic screening in asymptomatic women at risk for ovarian cancer. Gynecol Oncol. 2000;77:350Y356.

22. Varras M. Benefits and limitations of ultrasonographic evaluation of uterine adnexal lesions in early detection of ovarian cancer. Clin Exp Obstet Gynecol. 2004;31:85-98.

23. Geomini PM, Kluivers KB, Moret E, Bremer GL, Kruitwagen RF, Mol BW. Evaluation of adnexal masses with three-dimensional ultrasonography. Obstet Gynecol. 2006;108:1167-1175.

24. Alcázar JL, Castillo G. Comparison of 2-dimensional and 3-dimensional power-Doppler imaging in complex adnexal masses for the prediction of ovarian cancer. Am J Obstet Gynecol. 2005;192:807-812.

25. Kaijser J, Sayasneh A, Van Hoorde K, et al. Presurgical diagnosis of adnexal tumours using mathematical models and scoring systems: a systematic review and meta-analysis. Hum Reprod Update. 2014;20: $449-462$.

26. Valentin L, Hagen B, Tingulstad S, Eik-Nes S. Comparison of 'pattern recognition' and logistic regression models for discrimination between benign and malignant pelvic masses: a prospective cross validation. Ultrasound Obstet Gynecol. 2001;18:357-365.

27. Dodge JE, Covens AL, Lacchetti C, et al. Preoperative identification of a suspicious adnexal mass: a systematic review and meta-analysis. Gynecol Oncol. 2012;126(1):157-166.

28. Sokalska A, Timmerman D, Testa AC, et al. Diagnostic accuracy of transvaginal ultrasound examination for assigning a specific diagnosis to adnexal masses. Ultrasound Obstet Gynecol. 2009;34: $462-470$.
International Journal of Women's Health

\section{Publish your work in this journal}

The International Journal of Women's Health is an international, peerreviewed open-access journal publishing original research, reports, editorials, reviews and commentaries on all aspects of women's healthcare including gynecology, obstetrics, and breast cancer. The manuscript management system is completely online and includes

\section{Dovepress}

a very quick and fair peer-review system, which is all easy to use. Visit http://www.dovepress.com/testimonials.php to read real quotes from published authors. 